\title{
Recuerdo histórico del antiguo Servicio de Infecciosos del Hospital de Niños Manuel Arriarán
}

\author{
Enrique Laval R.
}

\section{Historical notes about the antique Infectious Diseases Unit at the Hospital de Niños Manuel Arriarán}

Measles and pertussis epidemics that occurred in Chile at the beginning of XX Century definitively motivated Government authorities to put up hospitals dedicated to infancy attendance. At first it was the hospital settled at Matucana Street (Roberto del Río) and afterwards since 1913, the Manuel Arriarán Hospital in the southern-east districts of Santiago. In this last one it was located the first Infectious Diseases ward organized in Chile, opened in 1922 and built according to most recent isolation and aseptic technics, projected by the proper Sub-Administrator of Manuel Arriarán Hospital, Professor Alejandro del Río MD, taking place early to the system that years later should be implemented at the Claude Bernard Infectious Diseases Hospital in Paris and at Hospital de Enfermedades Infecciosas Prof. Dr. Lucio Córdova at Santiago, Chile. Doctors Alfredo Commentz MD and Eugenio Cienfuegos MD, Sub-Director and Chief of Internal Medicina Department in Manuel Arriarán Hospital, respectively, had a relevant role in making rules and the development of this department. Distinguished faculties were members of the Infectious Diseases Department, standing out Professor Adalberto Steeger, an important promoter of Pediatric Infectious Diseases speciality in Chile. Proximately 30 years after, the Infectious Diseases Department was substituted by the Infectious Diseases Clinical Units attached to Internal Medicine Departments A and B at the same hospital.

Key words: Children hospital, history, infectious diseases.

Palabras clave: Hospital de niños, historia, enfermedades infecciosas.
Fundación del Hospital Clínico de Niños Manuel Arriarán

$\mathrm{L}$ a Junta Central de Beneficiencia, en sesión del 18 de octubre de 1910, acordó construir un Hospital de Niños, en la zona Sur-Oriente de Santiago. Para ello adquirió la Quinta "El Mirador" de la sucesión de don Eduardo Matte. ${ }^{1}$

El lugar elegido se encontraba ubicado en la calle Santa Rosa, en las proximidades de la Avenida Matta. ${ }^{2}$ Se acordó dar el nombre de Manuel Arriarán al nuevo establecimiento, ya que se destinaron cuatrocientos de los ochocientos mil pesos que este gran filántropo había legado, con dicho objeto, a la Beneficencia Pública. Para su fundación, el supremo Gobierno decretó, con fecha 15 de julio de 1911, la constitución de una comisión compuesta por los señores Ventura Blanco, Alejandro Hunneus y el doctor don Alejandro del Río, quienes redactarían la reglamentación del hospital ${ }^{1}$.

Como Administrador se designó a don Germán Riesco Errázuriz, quien exigió como condición indis- pensable para aceptar el cargo, que la Sub-Administración recayera en el doctor del Río, el que, a pesar de la intensa actividad que desarrollaba en el ejercicio pro-

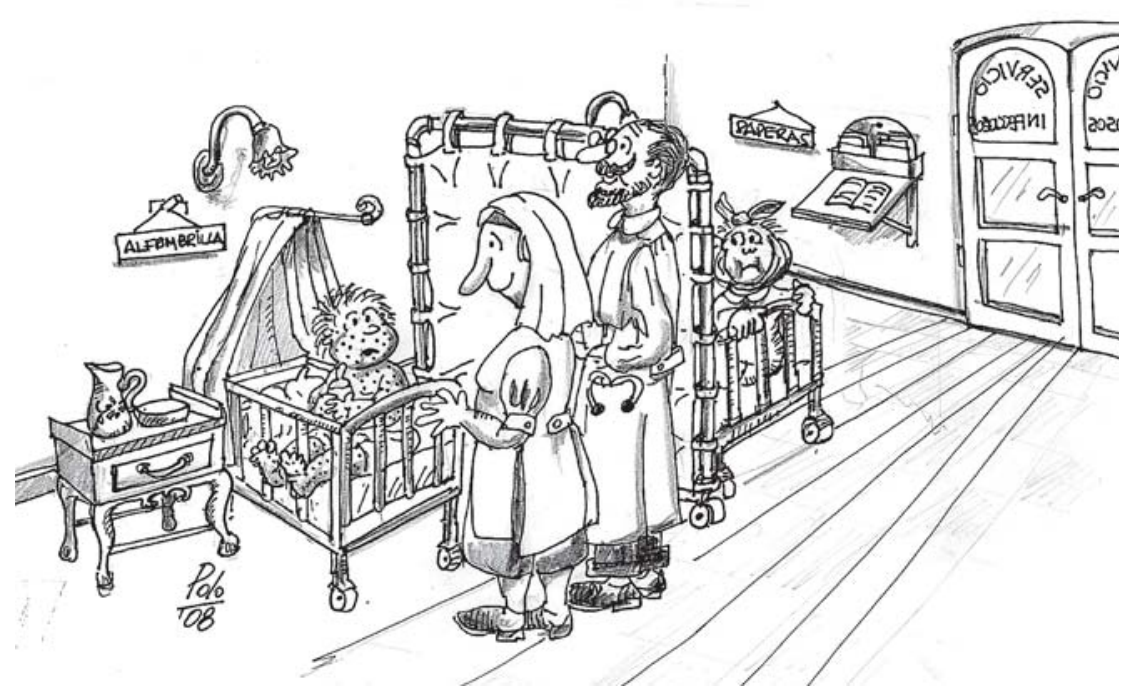



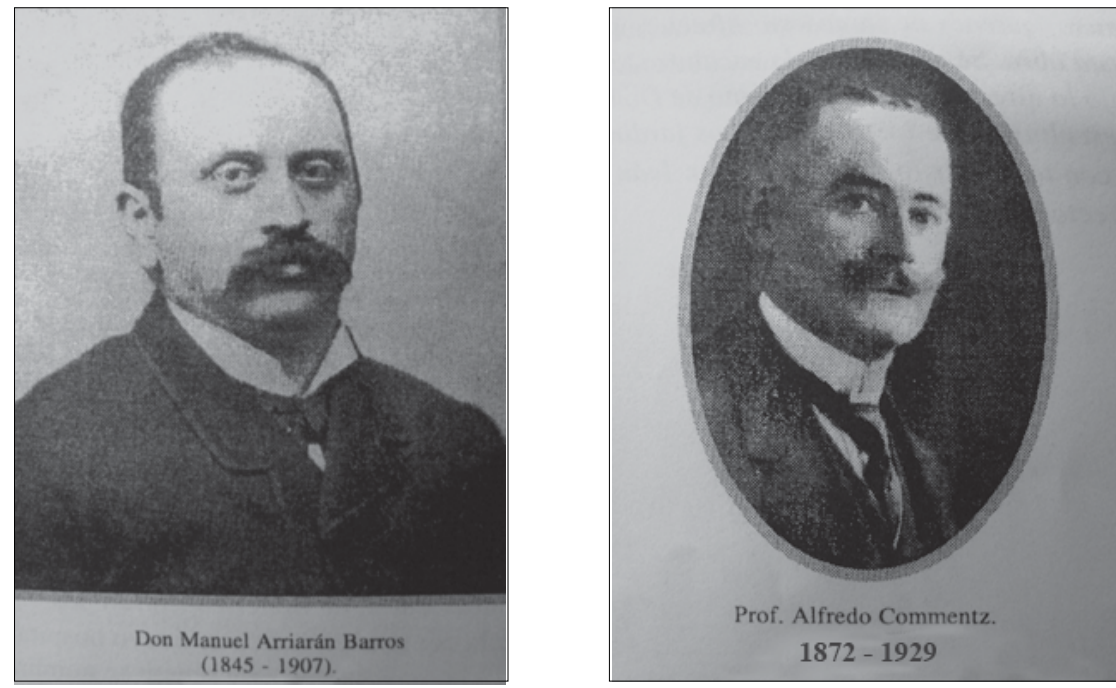

fesional, en la docencia y en la Asistencia Pública, aceptó compartirla con el hospital recién proyecta$\mathrm{do}^{2}$.

Don Manuel Arriarán Barros era un distinguido miembro de la Junta Central de Beneficencia, que había desempeñado con brillo la administración de lazaretos durante epidemias de viruela, actuando como reformador del Servicio de Vacunas. Activo y celoso Administrador del Cementerio General por más de 10 años. Luego, hasta su muerte el 15 de septiembre de 1907, Administrador del Hospital de Niños de la calle Matucana (después llamado Roberto del Río) ${ }^{3}$.

El proyecto arquitectónico fue encargado al señor Emilio Jecquier, secundado por el eminente pediatra doctor don Alfredo Commentz, diseñándose primitivamente un establecimiento con capacidad para 374 camas, distribuidas de la siguiente manera: Medicina 162, Infecciosos 50, Cirugía 115 y Lactantes 46

El hospital abrió sus puertas al público, el día $1^{\circ} \mathrm{de}$ enero de 1913, instalado en un departamento del antiguo edificio de la Quinta y reduciendo su acción a la atención de policlínico ${ }^{1}$. Sólo se pudo iniciar la internación de enfermos en 1919, necesidad cada vez más apremiante por el aumento de las consultas ${ }^{4}$

El programa de construcción de este hospital, dada la magnitud y el elevado costo de la obra, no logró realizarse en todo su conjunto ni a un mismo tiempo. Así, los edificios antiguos de la Quinta "El Mirador", convenientemente reparados, se utilizaron en forma provisoria para dar cabida a 100 camas, mientras se terminara la edificación de todas las nuevas construcciones que rodean el parque del predio.
La construcción del Servicio de Infecciosos. Desarrollo y reglamento

¿Qué se publicaba en el Anuario del Hospital Manuel Arriarán, del año 1919, sobre las enfermedades infecto-contagiosas?: "el programa de hospitalización reservado para los edificios antiguos del establecimiento, no consultaba ninguna disposición para la atención de los enfermos contagiosos, porque los pabellones definitivos aún no se construian. A pesar de todo, no se pudo prescindir de la admisión de enfermos de alfombrilla y coqueluche, porque las epidemias reinantes en aquella época, motivaron justamente la habilitación de este hospital. Nos encontramos frente a un problema serio: las salas no tenían disposiciones adecuadas para impedir la propagación de los contagios dentro del establecimiento conforme a las reglas de atención aséptica vigentes para tales casos. Fue menester dedicar un grupo de salas separadas del resto por un pasillo, a la atención de enfermedades contagiosas. Para los enfermos de alfombrilla, y tos convulsiva se aplicó el sistema de aislamiento en salas destinadas a cada uno de estos contagios. Para las demás enfermedades contagiosas, se usó el sistema de barreras, o sea el aislamiento del enfermo en sala abierta, por medio de divisiones de biombos. A este grupo de enfermedades pertenecian la fiebre tifoidea, tifus exantemático, escarlatina, difteria, influenza, erisipela, varicela, bronconeumonias sépticas, etc. Las medidas de aislamiento de barrera, han sido de tal suerte eficientes que la enfermedad ha podido ser concretada siempre a su portador original respectivo". "La alfombrilla es la única afección que presenta un evidente peligro de contagio dentro del establecimiento, aun cuando se pongan en uso todas las medidas profilácticas que tienden a evitarlo"s.

Entre los edificios que se construyeron en el Hospital Manuel Arriarán, a partir de 1920, estuvo el Servicio de Infecciosos, denominado "sub-sección", que constaba de cuatro pabellones, situados en el extremo suroriente del hospital, con entrada especial por la calle Santa Elvira. El primero, "Pabellón para el Personal" (llamado Pabellón Carmen y Dolores Arriarán, ambas hermanas de don Manuel), fue destinado a "recibir al personal compuesto de una Hermana, de ocho Enfermeras y de seis Auxiliares Administrativas", en situación de excepcionales condiciones sanitarias, para favorecer su resistencia contra el contagio y hacer menos penoso su forzado aislamiento del resto del personal residente

La sub-sección destinada a los enfermos infecciosos propiamente tales, comprendía un grupo de tres pabellones, de los cuales dos tenían dos pisos, con 


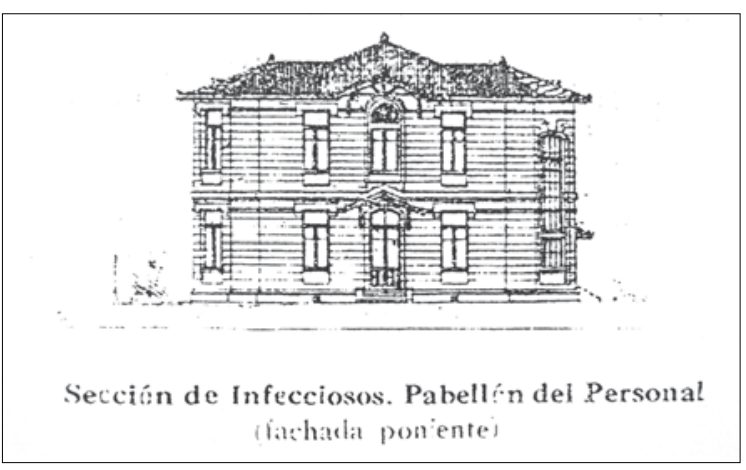

cabida para 33 enfermos cada uno y otro pequeño "pabellón-lazareto", para recibir a cuatro pacientes ${ }^{6}$.

La construcción del Servicio de Infecciosos se había iniciado a comienzos del año 1921, siendo inaugurado en septiembre del año 1922. Conviene señalar que todo esto fue proyectado personalmente por el doctor Alejandro del Río, quien se anticipó en su concepción al sistema que varios años después se implantaría en el Hospital de Enfermedades Infecciosas de París (Claude Bernard), el denominado "sistema de acordeón", que permitía en un mismo pabellón aislar enfermos de diversas afecciones contagiosas agudas, sin que entraran en contacto directo unos pacientes con otros y que daba margen para aumentar el número de camas de determinadas enfermedades, considerando siempre las normas fundamentales de aislamiento del enfermo y del personal. Este sistema se proyectaría, con algunas variaciones, en la década de 1940, en la construcción del Pabellón de Enfermedades Infecciosas del Hospital Ramón Barros Luco (desde 1963, Hospital de Enfermedades Infecciosas Profesor Dr. Lucio Córdova) ${ }^{2}$.

La distribución consultada para esta sub-sección, permitía efectuar el aislamiento absoluto de enfermedades con varios tipos de contagio. Los pabellones de dos pisos (Pabellón Petronila Salamanca y Manuel Orellana, ambos benefactores del Hospital), tenían entrada separada para cada piso, pudiendo ser manejados con total independencia unos de otros. En caso necesario, una puerta de comunicación interior permitía reunir los dos pisos para un solo grupo de enfermos. A su vez los pisos bajos de estos pabellones podían ser unidos aisladamente o en conjunto con el lazareto.

El pequeño pabellón de un piso, llamado "lazareto" (Pabellón Sor Juana de Jesús, Madre Superiora, en homenaje a su inteligente labor en este establecimiento), estaba destinado a recibir los primeros casos de una epidemia o algún contagio de carácter peligroso.

A cada una de las cuatro secciones se les había asignado un trozo de jardín, separado "uno de otro

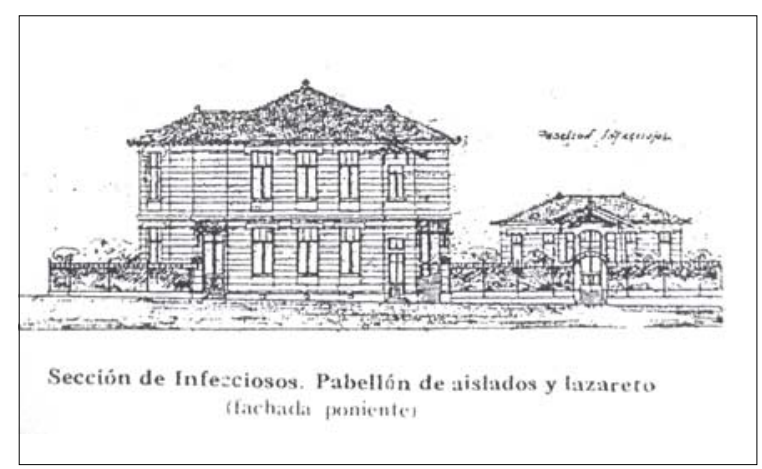

con cerco vivo de rosas, a fin de que los niños en convalecencia pudieran moverse al aire libre, recibiendo los beneficios del sol"6.

En 1923, en la Memoria del Servicio Médico, realizada por el doctor Alfredo Commentz, sub-director del Hospital Manuel Arriarán, se anota "que ha aumentado considerablemente el número de enfermos infecciosos, debido una vez más, a la reaparición de las epidemias de alfombrilla y tos convulsiva. El Servicio de Enfermedades Infecciosas, instalado hace poco tiempo en su local definitivo, ha despertado entre el personal médico el más vivo interés, por ser el primero en su género, establecido en forma cientifica en nuestro país. Han debido hacerse numerosas perfecciones en su manejo técnico, viéndose la Dirección del Hospital en la necesidad de establecer un Reglamento especial para esta Sección, conjuntamente con las instrucciones detalladas para mantener una profilaxis general y especial, con el fin de evitar hasta donde sea posible, las contaminaciones dentro del establecimiento ${ }^{17-9}$

El doctor Commentz manifestó sus sinceros agradecimientos al doctor Alejandro del Río, por la valiosa colaboración en la confección del Reglamento del Servicio de Infecciosos, el que llamó la atención por su claridad conceptual y prolijidad. En el Título I, abordaba reglas generales con objetivos muy precisos, como también la prevención de infecciones intra-hospitalarias que podían significar pérdidas de vidas con las consiguientes responsabilidades de los directivos.

Este Reglamento redactado exactamente hace 85 años, expone conceptos importantes sobre el manejo de las enfermedades infecciosas transmisibles, su prevención, técnicas de desinfección, métodos de profilaxis, tratamiento de basuras y desperdicios, etc, dedicados a los médicos y al personal hospitalario en general, en una época en que la Salud Pública estaba "muy en pañales"7.

En el Anuario del Hospital Manuel Arriarán, correspondiente al año 1925, se destaca que "el Servicio de 
Infecciosos ha marchado en forma correcta, anotándose en su estadistica respectiva de morbilidad, uno que otro caso de "infección doméstica", dentro del establecimiento". Pero, la mortalidad había aumentado en este Servicio, debido a dos circunstancias especiales: la producción de complicaciones bronco-pulmonares graves en los enfermos de tos convulsiva, así como la hospitalización de pacientes con bronconeumonías de otras etiologías, que han ocasionado 138 fallecidos, $31,1 \%$ de la mortalidad general del hospi$\operatorname{tal}^{10}$.

La otra circunstancia importante, digna de considerar, es que la atención de los enfermos tuberculosos se efectuó desde el comienzo en el Servicio de Infecciosos. La observación continuada de la modalidad de atención de estos enfermos, llevó a la convicción de que era necesario crear un Servicio de Tisiología con carácter independiente, debido a la gravedad que entrañaba para tales pacientes, su contagio con enfermedades infecciosas agudas. La Dirección del Hospital se dio a la tarea de buscarle una ubicación adecuada, efectuándose las reparaciones necesarias para lograr la instalación del Servicio de Tisiología, al que se le dio el nombre de Santa Margarita y que finalmente fue inaugurado a fines del año 1939.

Los administradores o directores del Hospital Manuel Arriarán, en los primeros casi 30 años de su funcionamiento, fueron: don Germán Riesco Errázuriz (1910-1916), don Ismael Valdés Valdés (1916-1927), don Juan Valdés Ortúzar (1927-1929), don Domingo Edwards Matte (1929-1931), doctor Julio Schwarzenberg Lobeck (1931), doctor Eugenio Cienfuegos Bravo (1931-1932), doctor Agustín Inostroza Pérez (1932-1938) y el doctor Iván Prieto Nieto, quien asumió en el año $1938 .^{1}$

\section{El equipo médico del Servicio de Infecciosos}

El Servicio de Infecciosos (o Sub-Sección) dependía jerárquicamente del Médico Jefe de la Sección de Medicina. Se menciona como Jefe del Servicio de Infecciosos, en el año 1926, al doctor Guillermo Lermanda, quien había ingresado al hospital en el año 1917, siendo además Médico Residente. En el año 1927 continuaba desempeñando el cargo de Jefe de Servicio. En 1933, asumió las funciones de jefatura el doctor Adalberto Steeger Sch., siendo ayudantes primeros la Dra. Faustina Casarino F., y el doctor Antonio Mattar M. Ayudantes segundos lo fueron la Dra. Otilia Morales Z. y el Dr. Rodulfo Philippi B. Ayudante adhonorem, el doctor Salvador Bustos. Al año siguiente, 1934, figura como Jefe, el doctor Guillermo Morales B., ayudante primero el doctor Agustín Fernández y ayu- dantes segundos, los doctores Jorge Peña C., Horacio Aguirre A. y Alfredo Dabancens L., quien escribió una monografía sobre varicela y herpes zoster. En el año 1935, no hubo cambios en el equipo médico del Servicio de Infecciosos.

El doctor Adalberto Steeger obtuvo en 1947 el título de Profesor Extraordinario de Clínica Pediátrica, con la tesis "Estudios clínicos de la difteria". Fue un brillante protagonista y gestor en todos los campos de la atención infantil y la docencia universitaria, trasladándose en abril de 1954 al Servicio de Pediatría del Dr. Julio Schwarzenberg, en el Hospital San Juan de Dios. Falleció el 23 de abril de 1973.

Con ocasión de la epidemia de sarampión habida en el invierno y primavera del año 1933, el Profesor Steeger tuvo la oportunidad de exponer, en una de las reuniones clínicas del Hospital Manuel Arriarán, las bases teóricas y prácticas sobre las cuales descansaba la profilaxis de esta enfermedad y las experiencias clínicas que sobre esta materia le fue posible recoger, en el Servicio de Infecciosos del Hospital Manuel Arriarán ${ }^{11-17}$.

\section{Los profesores doctores Alfredo Commentz Loefler y Eugenio Cienfuegos Bravo}

Es de toda justicia mencionar a dos médicos que tuvieron gran importancia en el desarrollo del Hospital Manuel Arriarán y por supuesto en la organización de su Servicio de Infecciosos. Ellos fueron el doctor Alfredo Commentz Loefler, nacido en Lima, Perú, en 1872, pero que a los 21 años tuvo la oportunidad, por acuerdo internacional de elegir nacionalidad, naturalizándose chileno. Se recibió de médico-cirujano en 1901. A su regreso de Alemania en 1913, donde efectuó estudios de perfeccionamiento, se preocupó intensamente de la fundación y desarrollo del Hospital Manuel Arriarán, siendo el colaborador más entusiasta del doctor Alejandro del Río y de don Germán Riesco, encargados por la Beneficencia de su construcción. Sus trabajos científicos en diversos tópicos de la Pediatría, además de las brillantes exposiciones de sus clases, le valieron en 1922 ser reconocido por la Facultad de Medicina de la Universidad de Chile, como Profesor Extraordinario de Pediatría, después de aprobar su tesis "Práctica dietética en los Hospitales".

Otras de sus importantes obras fueron la organización de la Escuela de Enfermería del Hospital Manuel Arriarán y el Reglamento para el Servicio de Infecciosos, que ya ha sido mencionado. En 1928, renunció a su cargo de Sub-Director del Hospital, siendo nombrado Médico Honorario de la Beneficencia Pública, falleciendo en 1929. 
Finalmente, el doctor Eugenio Cienfuegos Bravo, titulado de médico en 1908, fue durante largos años Jefe de la Sección Medicina y al que le correspondió la organización de toda la especialidad pediátrica del Hospital, ambulatoria al comienzo y de hospitalización posteriormente. Nombrado Profesor Extraordinario de Pediatría en 1926, además de excelente clínico, poseía una gran cultura humanística y era un amante del arte y de la literatura.

Dos años después de su muerte, en octubre de 1958, se inauguró en los jardines del Hospital, un busto que recuerda su persona, escultura que fue obra del Profesor Eduardo Keymer ${ }^{3}$.

Los años 1938 y 1939 marcaron otra época en el desarrollo del Hospital Manuel Arriarán, ya que mediante la obtención de fondos extraordinarios, se abordaron adaptaciones, reparaciones y transformaciones sustanciales. El servicio más inconexo del establecimiento era el de Otorrinolaringología: su consultorio externo ubicado en el policlínico general, donde se efectuaba la cirugía y con su sala de hospitalizados, en la sección de cirugía. Para instalarlo en debida forma, se dispuso del primer pabellón del Servicio de Infecciosos, aquel que fue destinado en un principio a albergar al personal de enfermeras y de auxiliares de servicio que atendía dicha sub-sección. En la planificación primitiva del hospital, dicho pabellón fue concebido con tal objeto con miras a la mejor profilaxis intra-hospitalaria; sin embargo, poco a poco, el edificio se transformó en el hogar de enfermeras generales del hospital, siendo necesario dar los primeros pasos hacia la institución del régimen de externado para las enfermeras. Previo a ciertas transformaciones, el pabellón que llevaba los nombres de Carmen y Dolores Arriarán, fue entregado el año 1939 al Servicio de Otorrinolaringología, disponiendo dentro de el, de las comodidades necesarias para la atención del consultorio externo, hospitalización, pensionado, del pabellón de operaciones, etc ${ }^{1}$.

El profesor doctor Francisco Mardones Restat, Director del Hospital Manuel Arriarán en la década del 60 , me comunicó que en aquella época y a raíz de un grave brote de sarampión, se vio obligado a solicitar al Médico jefe del Servicio de Otorrinolaringología, doctor Raúl Barrios, el traslado al subterráneo del Pabellón Carmen y Dolores Arriarán de su servicio, con el fin de instalar una Unidad de Urgencia para enfermos con complicaciones respiratorias obstructivas altas del sarampión, para lo cual proveyó de croupetes, respiradores, etc, gracias a donaciones recibidas desde los Estados Unidos de Norteamérica. También, más o menos, en el mismo período en que hubo alarmante incremento de enfermos con poliomielitis, cuya fase aguda fue atendida en el Servicio de Infecciosos, se estable- ció un convenio para la rehabilitación en su etapa paralítica, con el Hospital Susana Palma, ubicado en el Barrio Franklin de la capital ${ }^{18}$

El profesor doctor Arturo Baeza Goñi. El profesor doctor Julio Meneghello Rivera y su equipo. Reemplazo del Servicio de Infecciosos por Unidades Clínicas de Enfermedades Infecciosas

La labor del profesor Eugenio Cienfuegos, quien había sido designado Profesor Titular de Pediatría, en el año 1928, fue continuada por el doctor don Arturo Baeza Goñi, titulado en 1919, desempeñándose como Médico Residente del Hospital a partir de 1921, donde ejerció durante 43 años sin interrupción. Recibió su título de Profesor Extraordinario de Pediatría en el año 1943, con la memoria sobre "La glomerulonefritis en la infancia". En 1951, fue nombrado Profesor Titular de la especialidad, cuando ya ocupaba el cargo de jefe del Servicio de Medicina del Hospital Manuel Arriarán. "El sello que más distinguió al profesor Baeza Goñi fue su trabajo en Pediatría social".

A su cátedra y servicio llegaría como Profesor Extraordinario y jefe de Clínica, el doctor don Julio Meneghello Rivera, en 1949, si bien desde 1943, era ya médico del Hospital, Con un selecto grupo de ayudantes, produciría grandes cambios en la docencia y en el enfoque a todo nivel de la Pediatría. Tiempo después el Servicio de Medicina del Hospital Manuel Arriarán se dividiría en Servicio A, a cargo del profesor Meneghello y el B, con la jefatura del profesor Arturo Baeza Goñi.

Entre los colaboradores directos del doctor Julio Meneghello, destacaba nítidamente el profesor doctor Enrique Fanta Núñez, quien durante los años 1961 a 1963, se desempeñó como Supervisor de la Unidad de Infecciosos del Servicio A de Medicina. El médico argentino doctor Sixto Gerardo González, ex becado en la cátedra del profesor Meneghello, lo recuerda "dirigiendo la Unidad de Infectología, con un importante número de meningitis, en que se realizaban punciones lumbares y ventriculares diarias, neumopatias post-sarampionosas y estafilocócicas" ${ }^{4,19}$.

Con el correr del tiempo, el antiguo Servicio de Infecciosos del Hospital Manuel Arriarán había desaparecido. A partir de 1991, en el que fue Pabellón Carmen y Dolores Arriarán, comenzó a funcionar la Fundación Arriarán, a cargo del doctor Marcelo Wolff Reyes y de la doctora Rebeca Northland, para la atención y tratamiento ambulatorio de enfermos con SIDA $^{20}$.

Quizás, como final de este no bien hilvanado recuerdo del antiguo Servicio de Infecciosos del Hospi- 
tal Manuel Arriarán, convendría citar lo escrito por el doctor Pedro Martínez Saravia: "La idea de hospitalizar en forma especializada a estos enfermos infecciosos flota entre nosotros desde hace años. Algunos ensayos se han hecho: si no estoy mal informado la iniciación del Barros Luco, fue destinada a este objeto. Posteriormente en el hospital de Niños Manuel Arriarán ya se consultó francamente los pabellones aislados para algunas de estas enfermedades, con brillantes resultados ${ }^{\prime 21}$.

\section{Resumen}

Las epidemias de sarampión y coqueluche, ocurridas en Chile a comienzo del siglo XX, motivaron definitivamente a las autoridades gubernamentales, a construir hospitales para la atención de niños. Primero el hospital de la calle Matucana (Roberto del Río) y luego en la zona Sur-Oriente de Santiago, el Hospital Manuel Arriarán en 1913. En este último se ubicó el primer
Servicio de Infecciosos en Chile, inaugurado en 1922 , realizado de acuerdo con las técnicas más recientes de aislamiento y asepsia, proyectado por el propio SubAdministrador del Hospital Manuel Arriarán, profesor doctor Alejandro del Río, anticipándose al sistema que años después se llevaría a cabo en el Hospital de Enfermedades Infecciosas Claude Bernard de París y en el Hospital de Enfermedades Infecciosas Prof. Dr. Lucio Córdova de Santiago de Chile. Los doctores Alfredo Commentz y Eugenio Cienfuegos, Sub-Director y Jefe de la Sección Medicina del Hospital Manuel Arriarán, respectivamente, tuvieron una participación muy importante en la reglamentación y desarrollo de dicho Servicio. Distinguidos profesionales fueron miembros del equipo médico del Servicio de Infecciosos, sobresaliendo el profesor Adalberto Steeger, importante impulsor de la Infectología Pediátrica en el país. Al cabo de más o menos 30 años, el Servicio de Infecciosos fue reemplazado por Unidades Clínicas de Enfermedades Infecciosas adjuntas a los Servicios de Medicina A y B de dicho establecimiento.

\section{Referencias}

1.- Prieto, N. I. El Hospital Manuel Arriarán. (1910-1944). Rev Asist Soc 1944; 13: 311-9.

2.- Laval M E. Don Alejandro del Río. Rev Asist Soc 1944; 13: 135-270.

3.- Artigas N R, Montenegro O E. Origen y desarrollo de los hospitales para niños en Santiago. Hospital Manuel Arriarán. U. de Chile. Santiago de Chile. 2001; p. $57-75$

4.- Vargas C N. Historia de la Pediatría Chilena: Crónica de una alegría. Ed. Universitaria. 2002. Santiago de Chile.

5.- Las enfermedades infecto-contagiosas. Anuario del Hospital Manuel Arriarán. 1919

6.- Commentz A. El Hospital de Niños Manuel Arriarán. Ed. Universitaria. Santiago de Chile. 1924; p. 5-70.
7.- Commentz A. Reglamento de la Sub-Sección de Infecciosos del Hospital de Niños Manuel Arriarán. Anuario del Hospital Manuel Arriarán. 1923. Impta. y Lit. La Ilustración p.49-61. Santiago de Chile.

8.- Commentz A. Memoria del Servicio Médico. Anuario del Hospital Manuel Arriarán. 1929. Impta. y Lit. La Ilustración p. 20-22. Santiago de Chile.

9.- Artigas N R. Hospital Manuel Arriarán. Su historia. Rev Serv Salud Metrop Central 1991; 1: 21-3.

10.- Commentz A. Memoria del Servicio Médico. Anuario del Hospital Manuel Arriarán. 1925 ; p. 25-26.

11.- Anuario del Hospital Manuel Arriarán. Santiago de Chile. 1926.

12.- Anuario del Hospital Manuel Arriarán. Santiago de Chile. 1927.

13.- Archivos Clínicos del Hospital Manuel
Arriarán. Santiago de Chile. 1933

14.- Archivos Clínicos del Hospital Manuel Arriarán. Santiago de Chile. 1934

15.- Archivos Clínicos del Hospital Manuel Arriarán. Santiago de Chile. 1935.

16.- Steeger S A. Profilaxis del sarampión. Reunión Clínica. Hospital Manuel Arriarán. $11 / 10 / 1933$

17.- Velasco R C. Homenaje a Prof. Dr. Adalberto Steeger Schaeffer. Rev Chil Pediatr 1989; 60: 369-76.

18. - Mardones Restat F. Comunicación personal.

19.- González S G. Meneghello, su equipo y su obra. No figura editorial. Córdoba. Argentina. 2003.

20.- Wolff R M. Comunicación personal.

21.- Martínez P. Algunas consideraciones sobre hospitalización racional de infectocontagiosos en Chile. Rev Asist Soc 1940; 9: 236-46. 